\title{
Glycosyltransferases as Potential Drug Targets
}

\section{Xue-Long Sun*}

Department of Chemistry, Chemical and Biomedical Engineering, Cleveland State University, Cleveland, OH 44115, USA

Glycoconjugates, existing as glycoproteins, glycolipids and proteoglycans on cell surface, are involved in many biological processes, such as cell-cell communication, signal transduction, immune response, and microbial adhesion and infection [1]. Structures features of glycoconjugates are vital in specific biological processes, however, change dramatically during disease development such as malignant transformation [2]. Glycosyltransferases (GTs) manage the biosynthesis of glycans and glycoconjugates, and thus play essential roles in these biological processes [3]. Therefore, intervention of GTs has attracted remarkable interest for drug development since inhibitors of GTs can potentially interfere pathological processes, in which the GTs are involved [4]. With the recent advances of glycomics and chemical glycobiology, the considerable potential of GTs as drug targets has been revealed and prospective strategy for GTs inhibition have been developed, especially in therapeutic areas of inflammation, cancer and infection diseases. This Editorial highlights the recent advances in drug development targeting GTs.

In general, GTs transfer sugar nucleotide donors onto suitable acceptors for glycans and glycoconjugates biosyntheses [5]. Both donor and acceptor substrates are recognized by GTs binding pockets. Therefore, donor, acceptor and bisubstrate analogues have been proposed as potent inhibitors of GTs [6]. It is common to modify the sugar moiety of donor nucleotide analogues for developing GTs inhibitors. Pesnot et al. recently reported a strategy by modifying the base moiety of donor nucleotide for GT inhibitor development [7]. The authors found that 5-substituted UDP-Gal derivatives bind the Leloirtype galactosyltransferases (GalTs) just as well as the natural donor but inhibit the GalTs catalytic activity potentially. In this study, they demonstrated that the 5-formylthien-2-yl group blocked a significant conformational change upon substrate binding and locked the enzyme in an unproductive conformation, and thus inhibit the GalTs activity. In addition, they found that this strategy works against other GTs [8]. The strategy aimed to modify the base moiety of donor nucleotide may provide a valuable way to develop potent GT donor analogues inhibitors.

It has been recognized that most donor nucleotide analogues have poor membrane permeability and thus may have poor pharmacokinetic property in vivo due to their highly hydrophilic and negative charge nature. Recent chemical glycobiology advances verified that GT donor nucleotide biosynthetic pathways could tolerate various sugar analogues of nature for the biosynthesis of glycan structures within cells [9]. Therefore, these pathways could be exploited in an alternative way to generate GT inhibitors. Gloster et al. first demonstrated that unnatural sugar precursor could be used as potential GT inhibitors since it could be metabolically converted to the corresponding sugar nucleotide donor analog by the cell and then inhibit GT as it bound but not used as a substrate by the enzyme [10]. By taking the metabolic advantage of the relaxed substrate specificity of enzymes in the sialic acid and fucose salvage pathways, recently, Rillahan et al. reported that sialic acid and fucose analogs could serve as selective sialyltransferase and fucosyltransferase inhibitors [11]. In this study, they showed that peracetylated sialic acid and fucose bearing a fluorine atom proximal to the endocyclic oxygen are membrane-permeable and could be converted to the corresponding sugar nucleotide analogs intracellularly, and then effectively inhibit the synthesis of sialylated and fucosylated glycan epitopes. This study demonstrated that membrane permeable sialic acid or fucose analogues may have good pharmakokinetics and pharmakodynamics targeting GT enzymes that decorate glycans with sialic acid or fucose. It is known that sialyltransferases and fucosyltransferases are involved in oligosaccharide such as sialyl lewis X (SLeX) biosynthesis, which plays key roles in host-pathogen interactions and leukocyte trafficking [12]. Overexpression of SLeX is linked to chronic inflammation conditions and various cancers. Therefore, selective and effective inhibition of these enzymes provides a promising strategy for drug development for the treatment of inflammation and cancer disease. This study further indicated that GTtargeting metabolic inhibitory mechanism may provide a promising strategy for drug development.

On the other hand, cancer cells often express many aberrant glycoforms when compared with normal cells [2]. It is known that the expression of highly branched $\mathrm{N}$-glycans is distinctly enhanced during the proliferation of different cancer cells and metastasis [13]. Therefore, inhibitors and modifiers of the biosynthesis of such highly branched glycans would prevent the highly expression of the aberrant glycoforms and thus may become potential candidates for cancer therapy. Nishimura et al. reported a metabolic inhibitory mechanism on human prostate cancer cell proliferation by $\mathrm{N}$-acetylglucosamine (GlcNAc) derivatives [14]. Particularly, they found that per-O-acetylated GlcNAc with a fluorine atom at the $\mathrm{C} 4$ position is a precursor of the metabolic inhibitor of human cancer cell proliferation, and showed higher inhibitory effect on PC-3 cell growth than that of 5-fluorouracil or cisplatin. This study was another example to use metabolic inhibitory mechanism as a potential drug development approach targeting GTs.

Overall, GTs plays key roles in many biological processes of human health and disease, such as inflammation, cancer and infection diseases. Therefore, GTs are potential drug targets in a range of therapeutic areas. GT inhibitors are potential lead compounds for drug discovery. Conventional donor nucleotide analogues have been proposed as inhibitors of GTs but have less chance to be developed as drug candidates due to their poor membrane permeability. Recently, metabolic inhibitors of GTs show great potential to modulate glycosylation and thus present a promising platform for developing drugs for various diseases. Continued advances in glycobiology and glycomics study will uncover the physiological and pathological roles of various glycoconjugates and related glycan processing-enzymes such as GTs and thus will provide tremendous opportunity for drug development. Particularly, understanding of the functions of glycoconjugates and the mechanism of glycan-processing enzymes in pathological pathways will be crucial for validating new potential

${ }^{*}$ Corresponding author: Xue-Long Sun, Department of Chemistry, Chemical and Biomedical Engineering, Cleveland State University, Cleveland, OH 44115, USA Tel: 1-(216)687-3919; E-mail: x.sun55@csuohio.edu

Received February 22, 2013; Accepted February 23, 2013; Published February 25, 2013

Citation: Sun XL (2013) Glycosyltransferases as Potential Drug Targets. Med chem 3: e106. doi:10.4172/2161-0444.1000e106

Copyright: (c) 2013 Sun XL. This is an open-access article distributed under the terms of the Creative Commons Attribution License, which permits unrestricted use, distribution, and reproduction in any medium, provided the original author and source are credited. 
therapeutic GT targets and eventually discovering new GT inhibitorbased drugs for therapeutic applications.

\section{References}

1. Varki A, Cummings RD, Esko JD, Freeze HH, Stanley $P$ (2009) Essentials of Glycobiology, (2ndedn), Cold Spring Harbor (NY): Cold Spring Harbor Laboratory Press.

2. Hakomori S (2002) Glycosylation defining cancer malignancy: new wine in an old bottle. Proc Natl Acad Sci USA 99: 10231-10233.

3. Gloster TM, Vocadlo DJ (2012) Developing inhibitors of glycan processing enzymes as tools for enabling glycobiology. Nat Chem Biol 8: 683-694.

4. Wagner GK, Pesnot $T$ (2010) Glycosyltransferases and their assays. ChemBioChem 11: 1939-1949.

5. Breton C, Fournel-Gigleux S, Palcic MM (2012) Recent structures, evolution and mechanisms of glycosyltransferases. Curr Opin Struct Biol 22: 540-549.

6. Izumi M, Yuasa $H$, Hashimoto $H$ (2009) Bisubstrate analogues as glycosyltransferase inhibitors. Curr Top Med Chem 9: 87-105.

7. Pesnot T, Jørgensen R, Palcic MM, Wagner GK (2009) Structural and mechanistic basis for a new mode of glycosyltransferase inhibition. Nat Chem Biol 6: 321-323.
8. Descroix K, Pesnot T, Yoshimura Y, Gehrke SS, Wakarchuk W, et al. (2012) Inhibition of galactosyltransferases by a novel class of donor analogues. J Med Chem 55: 2015-2024.

9. Agard NJ, Bertozzi CR (2009) Chemical approaches to perturb, profile, and perceive glycans. Acc Chem Res 42: 788-797.

10. Gloster TM, Zandberg WF, Heinonen JE, Shen DL, Deng L, et al. (2011) Hijacking a biosynthetic pathway yields a glycosyltransferase inhibitor within cells. Nat Chem Biol 7: 174-181.

11. Rillahan CD, Antonopoulos A, Lefort CT, Sonon R, Azadi P, et al. (2012) Global metabolic inhibitors of sialyl- and fucosyl transferases remodel the glycome. Nat Chem Biol 8: 661-668.

12. Varki A (2007) Glycan-based interactions involving vertebrate sialic-acidrecognizing proteins. Nature 446: 1023-1029.

13. Dennis JW, Laferté S, Waghorne C, Breitman ML, Kerbel RS (1987) Beta 1-6 branching of Asn-linked oligosaccharides is directly associated with metastasis. Science 236: 582-585

14. Nishimura S, Hato M, Hyugaji S, Feng F, Amano M (2012) Glycomics for drug discovery: metabolic perturbation in androgen-independent prostate cancer cells induced by unnatural hexosamine mimics. Angew Chem Int Ed Engl 51: 3386-3390. 